\title{
Three patients with intracranial tuberculomas with unusual features
}

\author{
D. C. THRUSH AND D. D. BARWICK \\ From the Regional Neurological Centre, Newcastle General Hospital, Newcastle upon Tyne
}

SYNOPSIS Three patients with verified intracranial tuberculomas are reported. One patient presented with attacks of hydrocephalus initially diagnosed erroneously as migraine, another had multiple tuberculomas and a fourth tuberculoma became apparent during treatment with antituberculous therapy. The third patient presented with a frontal lobe syndrome and the postoperative recovery was complicated by the development of hyperosmolar non-ketotic, non-acidotic diabetic pre-coma. Although there are no pathognomonic symptoms, signs, or radiological appearances of intracranial tuberculomas, a high index of suspicion should always be entertained during the investigation of non-European immigrants.

Before 1900 tuberculomas constituted a third of all intracranial tumours and more than $50 \%$ of intracranial tumours in children (Starr, 1889). In 1933 a survey of postmortem records (Garland and Armitage, 1933) revealed that $34 \%$ of all cerebral tumours were tuberculomas. By 1940 an analysis of 2,190 brain tumours collected from recent reviews (Wilson, 1940) showed that only $3.6 \%$ were tuberculomas and a more recent review (Maurice-Williams, 1972) demonstrated an incidence of $0.15 \%$ among 2,200 intracranial tumours seen at the Guy's-Maudsley neurosurgical unit from 1951-1972.

In 1970 Kocen and Parsons (1970) drew attention to some unusual neurological complications of tuberculosis, particularly in nonEuropean immigrants, though none of their patients had intracranial tuberculomas. We wish to report three patients with verified intracranial tuberculomas who have presented to this unit in the past 12 months.

CASE 1

J.A., a 28 year old Indian engineer from Tanzania, had lived in England for five years. At the age of 26 years he had a dry cough which lasted for two weeks and a routine radiograph of the chest showed right hilar lymphadenopathy. He received no specific treatment and a chest radiograph five months later was normal. Thereafter he remained well until five months before admission when he developed intermittent severe occipital headaches which on occasions were associated with teichopsia in the right homonymous visual field, nausea, and neck stiffness. Apart from a recent febrile illness, his general health was good. There was no relevant past medical or family history.

On admission to a local general hospital he was pyrexial $\left(38.2^{\circ} \mathrm{C}\right)$, but otherwise examination was normal. Routine haematological and biochemical investigations were normal apart from a raised erythrocyte sedimentation rate of $40 \mathrm{~mm}$ in one hour. Examination of the cerebrospinal fluid and radiographs of the chest and skull were normal. The pyrexia and headaches continued and he was transferred to the Regional Neurological Centre, Newcastle upon Tyne. Apart from mild discomfort on extension of the neck, neurological examination was normal. He continued to show a regular evening pyrexia and seven days after admission he suddenly became unconscious and apnoeic and required artificial ventilation. The right pupil was pinpoint and the left normal in size but unreactive to light. After 15 minutes he regained consciousness, his pupillary reflexes returned but both fundi showed papilloedema and haemorrhages. An emergency pneumoventriculogram showed equal and marked dilatation of both lateral and the third ventricles and a space occupying lesion in the vermis of the cerebellum.

At operation (Professor Hankinson) a small firm irregular tumour was removed from the vermis. Histological examination revealed a necrotizing epi- 
thelial and giant cell granuloma from which $\mathrm{Myco-}$ bacterium tuberculosis was cultured. The patient was treated with dexamethasone, para-aminosalicylic acid, isoniazid, and streptomycin and made a satisfactory recovery.

\section{CASE 2}

B.S., aged 16 years, was born in Newcastle upon Tyne of Asian parents. Shortly after the patient's birth her father developed pulmonary tuberculosis and she was given BCG vaccination. The father's tuberculosis recurred in 1970, though during this time the patient received no chemotherapy. Her mother and one sister also had a history of previous tuberculous infection.

The patient was in good health until March 1971 when she developed anorexia, nausea, night sweats, and a non-productive cough. She was admitted to Newcastle General Hospital and a diagnosis of miliary tuberculosis was made and this was confirmed bacteriologically.

Treatment was commenced with streptomycin $0.75 \mathrm{~g}$ daily, isoniazid $300 \mathrm{mg}$ daily, and ethambutol $1 \mathrm{~g}$ daily with progressive improvement in her symptoms and in the appearance of the chest radiograph. After three months the streptomycin was stopped. She received the remaining two drugs regularly for nine months but during two subsequent months the antituberculous therapy was taken irregularly. At this time she developed generalized throbbing headaches and experienced an isolated episode of loss of consciousness. On recovery neurological examination was normal. An electroencephalogram showed no localizing features and examination of the cerebrospinal fluid revealed no abnormalities. During the following two months the headaches increased in severity and one week before admission to the Regional Neurological Centre she became aware of intermittent diplopia.

On examination the patient was apyrexial. She had bilateral papilloedema with haemorrhages and two choroidal tubercles in the right fundus but otherwise examination was normal.

A full blood count and routine biochemical tests were normal apart from an erythrocyte sedimentation rate of $30 \mathrm{~mm}$ in the first hour. A radiograph of the chest revealed no abnormality but that of the skull showed demineralization of the dorsum sellae and clinoid processes. A ${ }^{99}$ technetium brain scan demonstrated three areas of increased uptake in the left temporal, parietal, and parieto-occipital regions. An electroencephalogram was diffusely abnormal and dominated by persistent underlying delta activity and a left carotid angiogram showed displacement of the anterior cerebral artery and internal cerebral veins to the right by 8 and $7 \mathrm{~mm}$ respectively.

A diagnosis of intracranial tuberculomas was made and she was treated with dexamethasone, streptomycin, isoniazid, and rifampicin with progressive improvement of her headaches and the fundal appearances.

Three months later the patient was readmitted with a six day history of severe right hemicranial headaches. On examination she was apyrexial. There was tenderness to percussion over the right parietal region, mild neck stiffness, and bilateral papilloedema. The choroidal tubercles were still evident in the right fundus, but otherwise examination was normal. A ${ }^{99}$ technetium brain scan now showed a fourth area of abnormal activity in the right parieto-occipital region. The antituberculous therapy was changed to pyrazinamide $1 \mathrm{~g}$ twice daily, prothionomide $500 \mathrm{mg}$ twice daily, isoniazid $600 \mathrm{mg}$ daily, ethambutol $1,200 \mathrm{mg}$ daily, and rifampicin $600 \mathrm{mg}$ daily and, in view of the progressive nature of the disease, it was decided that surgical intervention was necessary to obtain tuberculous material for culture and sensitivity studies. At operation (Mr. Kalbag) three tuberculomas were found in the left cerebral hemisphere, on one of which a biopsy was carried out. Acid-fast bacilli were seen on direct microscopy and histological examination showed a necrotizing granuloma. Unfortunately, no organisms could be cultured and guinea-pig inoculation was negative.

The above antituberculous regime was continued for three months when it was reduced to rifampicin and isoniazid. During this period she complained of listlessness, difficulty in concentration, impairment of recent memory, and depression. Although these symptoms have slowly improved it has been impossible to wean her from the dexamethasone as a reduction below a dosage of $1 \mathrm{mg}$ daily resulted in headaches and papilloedema.

\section{CASE 3}

C.K., a 42 year old Indian lady who has lived in Great Britain for six years, was referred to a general hospital for investigation of a persistent vaginal discharge. Histological examination of a cervical biopsy revealed chronic granulomatous tissue and $\mathrm{Myco}$ bacterium tuberculosis was cultured from the biopsy material. Treatment was commenced with streptomycin, isoniazid, and rifampicin. During this time it was noted that her behaviour was odd, a radiograph of the skull revealed erosion of the pituitary fossa and she was transferred to the Regional Neurological Centre.

The patient spoke no English but from her relatives 
we learnt that there had been progressive deterioration in her personality and memory during the previous two years: she had been acting strangely and on occasions had been found wandering in the streets by the police. She had complained of intermittent frontal headaches which in recent months had become more severe and during the previous four weeks had become confused and disorientated. There was a vague history of visual deterioration and unsteadiness on walking, but no symptoms suggestive of pituitary failure. There was no relevant past medical history or family history.

On examination she was drowsy but rousable though cooperation was poor. There was no papilloedema or optic atrophy, the uncorrected visual acuity was $6 / 24$ bilaterally but because of the language barrier and personality change the visual fields could not be charted. The other cranial nerves were normal. Tone, power, and coordination of the limbs was normal but there was slight reflex dominance in the left lower limb associated with an equivocal plantar response. There was no apparent sensory deficit.

Normal investigations included a full blood count and erythrocyte sedimentation rate, serum urea and electrolytes, calcium, phosphate, serum transaminases, and thyroid function tests. A glucose tolerance test showed a latent diabetic curve. Examination of the cerebrospinal fluid revealed one white cell per cu $\mathrm{mm}$ and $50 \mathrm{mg} / 100 \mathrm{ml}$. of protein. The chest radiograph showed diffuse pulmonary shadowing in a peribronchial distribution above the right hilum and that of the skull enlargement of the pituitary fossa with complete destruction of the dorsum sellae and posterior clinoid processes. A ${ }^{99}$ technetium brain scan revealed an area of increased uptake to the right of the midline in the suprasellar region and bilateral carotid angiography confirmed the presence of a large suprasellar space occupying lesion which had totally obstructed the right anterior cerebral artery and partially obstructed the left. A pneumoventriculogram demonstrated a tumour lying in the region of the genu of the corpus callosum, anterior margin of the foramen of Monro, and lamina terminalis, which was causing partial obstruction of the foramen.

At operation (Professor Hankinson) the pole of the right frontal lobe was removed to expose a yellow, firm, lobulated tumour, $5 \mathrm{~cm}$ in diameter, which originated from a small area on the right side of the falx. The macroscopic appearance was suggestive of a meningioma but histological examination showed that the bulk of the tumour was acellular and necrotic and surrounded by tuberculous granulation tissue. In Zeil-Neilson stained sections a small number of acid-fast bacilli were found.
The patient made an uneventful immediate postoperative recovery, but remained confused, dis- $\frac{\mathscr{C}}{\circ}$ orientated and incontinent. Blood glucose estima- on tions were initially normal but three weeks after the operation she became drowsy and the biochemical 0

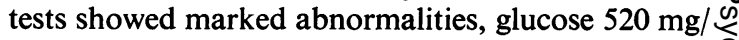
$100 \mathrm{ml}$., sodium $174 \mathrm{mg} / \mathrm{l}$., potassium $3.5 \mathrm{mEq} / \mathrm{l}$., 号 chloride $127 \mathrm{mEq} / \mathrm{l}$., bicarbonate $27 \mathrm{mEq} / \mathrm{l}$, and $\stackrel{0}{\Rightarrow}$ urea $85 \mathrm{mEq} / \mathrm{l}$. A diagnosis of hyperosmolar, non-? ketotic, non-acidotic diabetic pre-coma was made $\overrightarrow{\vec{F}}$

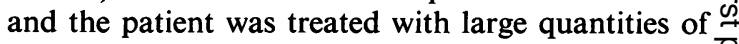
intravenous fluids and insulin with progressive improvement. Three months later, although she was $\frac{\bar{c}}{\bar{c}}$ fully mobile, there had been no change in her mental $\frac{\omega}{\sigma}$ state or incontinence. She required no hypoglycaemic $\propto$ agents and a glucose tolerance test showed a normal के response.

\section{DISCUSSION}

Although now rare in Great Britain, tuberculomas still represent a significant proportion of ? intracranial tumours in the world, varying from. $2.4 \%$ in Japan (Katsura et al., 1959) and $15 \%$ ig v Chile (Assenjo et al., 1951) to 30.5\% in Bomba (Dastur and Desai, 1965).

They may present with equal frequency in the supratentorial and infratentorial compartments and occur most frequently in the first three decades of life. Symptoms of raised intracrania $\vec{\theta}$ pressure are the usual presenting features but there are no specific symptoms or signs which distinguish them from other intracranial tumours (Sibley and O'Brien, 1956; Dastur and Desai, 1965). Tuberculosis elsewhere in the patient is a common finding (Dastur and Desai, 1965) though as many as $50 \%$ may have a negative chest radiograph. A family history of tuberculosis is variable but may be a useful diagnostic clue if present.

It is uncommon to see calcification of tuberculomas on plain skull radiographs, the incidence varying from $0-6 \%$ (Sibley and O'Brien, 1956; Ramamurthi and Varadarajan, 1961) and contrast radiology shows no diagnostic features. A sudden reduction in calibre of blood vessels traversing the tumour, such as occurred in case 3 , should arouse one's suspicions but is not pathognomonic (Dastur and Desai, 1965). Although angiography usually reveals an avascular space occupying lesion, subcortical tuberculomas may show a mild increased vascularity resembling that of low grade meningiomas (Ramamurthi 
and Varadarajan, 1961). Brain scanning is a comparatively recent diagnostic technique used in the investigation of cerebral pathology and for this reason has not been available in those countries where tuberculomas are most common. Cases 2 and 3 showed a markedly abnormal uptake of the isotope, presumably due to surrounding oedema and local disturbance of the blood-brain barrier, and scanning may prove to be a useful screening procedure. It is important to realize that the presence of multiple areas of increased uptake should not always lead to the conclusion that the patient has multiple metastases (see case 2).

At operation a tuberculoma may resemble a low grade astrocytoma, a metastatic lesion or, as $50 \%$ may have dural adhesions (Dastur and Desai, 1965), a meningioma. This is of some importance at operation, for if this fact is not appreciated the specimen may be fixed in formalin and the opportunity of obtaining material for culture and sensitivity studies is lost. The incidence of multiple tuberculomas is variable; they are not mentioned by Dastur and Desai in their series of 114 verified tuberculomas but Castro and Lepe (1963) quote an incidence of $16 \%$ and Asenjo et al. (1951) found $32 \%$ of multiple tuberculomas in their material.

All three patients were Asians. Case 1 presented with intermittent attacks of hydrocephalus diagnosed erroneously as migraine. Case 2 presented with a classical history of raised intracranial pressure and her past medical history and family history suggested the probable diagnosis. An unusual feature was the development of a fourth tuberculoma while on adequate antituberculous therapy, though recurrences elsewhere in the body in patients on long-term therapy have been reported previously (Dastur and Desai, 1965). As the patient was in hospital when this fourth lesion developed, she was known to have received regular antituberculous medication and drug resistance was therefore thought to be the probable explanation. However, a change in the antituberculous therapy produced little improvement. Although the patient has symptomatically improved, the brain scan 12 months later still demonstrated four areas of abnormal uptake and on each occasion that the dexamethasone has been withdrawn the headaches returned and papilloedema became evident. The explanation of this is not clear but benign intracranial hypertension secondary to long-term steroids appears unlikely. The confusion, personality change, and progressive dementia in the third patient were undoubtedly related to interference with the blood supply to the frontal lobes. The hyperosmolar, non-ketotic, nonacidotic diabetic pre-coma which complicated postoperative recovery was presumably precipitated by surgery though we have not encountered this before.

A leader writer in the Lancet (1970) wrote 'Tuberculosis of the nervous system has become so uncommon in the United Kingdom that it is rarely the first diagnosis that comes to mind'. While we agree with this, we feel that a high index of suspicion should always be entertained, particularly when investigating non-European immigrant patients and those with a past medical history of family history of tuberculosis.

We should like to thank Dr. J. B. Foster for allowing us to report on case 1 .

\section{REFERENCES}

Asenjo, A., Valladares, H., and Fierro, J. (1951). Tuberculomas of the brain. Archives of Neurology and Psychiatry (Chic.), 65, 146-160.

Castro, M., and Lepe, A. (1963). Cerebral tuberculoma. Acta Radiologica. Diagnosis, 1, 821-827.

Dastur, H. M., and Desai, A. D. (1965). A comparative study of brain tuberculomas and gliomas based upon 107 case records of each. Brain, 88, 375-396.

Garland, H. G., and Armitage, G. (1933). Intracranial tuberculoma. Journal of Pathology and Bacteriology, 37, 461-471.

Katsura, S., Suzuki, J., and Wada, T. (1959). A statistical study of brain tumors in neurosurgical clinics in Japan. Journal of Neurosurgery, 16, 570-580.

Kocen, R. S., and Parsons, M. (1970). Neurological complications of tuberculosis. Quarterly Journal of Medicine, 39, 1730.

Maurice-Williams, R. S. (1972). Tuberculomas of the brain in Britain. Postgraduate Medical Journal, 48, 678-681.

Neurological complications of tuberculosis (1970). Lancet, 1, 1094-1095.

Ramamurthi, B., and Varadarajan, M. G. (1961). Diagnosis of tuberculomas of the brain. Clinical and radiological correlation. Journal of Neurosurgery, 18, 1-7.

Sibley, W. A., and O'Brien, J. L. (1956). Intracranial tuberculomas. A review of clinical features and treatment. Neurology (Minneap.), 6, 157-165.

Starr, M. A. (1889). Tumours of the brain in childhood. Their variety and situation, with special reference to their treatment by surgical interference. Medical News, 54, 29-37.

Wilson, S. A. K. (1940). Neurology, 1, 593-596. Edited by A. N. Bruce. Arnold: London. 